\title{
Optimal Network Defense Strategy Selection Method: A Stochastic Differential Game Model
}

\author{
Yan Mi $\mathbb{D}^{1,2}$ Hengwei Zhang $\mathbb{D}^{1,2}$ Hao Hu $\mathbb{D}^{1,2}{ }^{1,2}$ Jinglei Tan $\mathbb{D}^{1,2}$ and Jindong Wang $\mathbb{D}^{1,2}$ \\ ${ }^{1}$ State Key Laboratory of Mathematical Engineering and Advanced Computing, Zhengzhou 450001, China \\ ${ }^{2}$ Zhengzhou Information Science and Technology Institute, Zhengzhou 450001, China \\ Correspondence should be addressed to Hengwei Zhang; wlby_zzmy_henan@163.com
}

Received 20 January 2021; Revised 6 July 2021; Accepted 30 July 2021; Published 24 August 2021

Academic Editor: Qing Yang

Copyright ( 2021 Yan Mi et al. This is an open access article distributed under the Creative Commons Attribution License, which permits unrestricted use, distribution, and reproduction in any medium, provided the original work is properly cited.

In a real-world network confrontation process, attack and defense actions change rapidly and continuously. The network environment is complex and dynamically random. Therefore, attack and defense strategies are inevitably subject to random disturbances during their execution, and the transition of the network security state is affected accordingly. In this paper, we construct a network security state transition model by referring to the epidemic evolution process, use Gaussian noise to describe random effects during the strategy execution, and introduce a random disturbance intensity factor to describe the degree of random effects. On this basis, we establish an attack-defense stochastic differential game model, propose a saddle point equilibrium solution method, and provide an algorithm to select the optimal defense strategy. Our method achieves real-time defense decision-making in network attack-defense scenarios with random disturbances and has better real-time performance and practicality than current methods. Results of a simulation experiment show that our model and algorithm are effective and feasible.

\section{Introduction}

With the rapid development of network information technology, network attack means emerge endlessly, and network security has a profound impact on people's daily life [1]. In 2019, more than 900 million network attacks were found in the world, 270 million URLs were identified as malicious URLs by web antivirus components, and $19.8 \%$ of computer users were attacked by malicious software at least once [2]. Network security is facing major threats and severe challenges. However, static defense technology represented by intrusion detection and antivirus software is difficult to cope with complex and changeable network attacks. How to analyze network attack behavior, deploy security defense system, and enhance system defense capability has become an urgent problem to be solved [3].

In selecting a defense strategy, we need to consider not only the effectiveness of the strategy, but also the cost constraints and attack strategies. Selecting the optimal defense strategy under the constraints of much complex information is the key to network defense [4]. As a decision- making theory in a conflict environment [5], game theory can help players select optimal strategies under complex conditions. In addition, with the basic characteristics of objective opposition, a noncooperative relationship, and strategic interdependence, game theory closely matches the fundamental characteristics of network attack and defense [6]. Therefore, the research of network attack and defense confrontation using game theory has become a hot spot in recent years and has achieved rich results [7-10].

In the process of attack and defense, the actions of the attacker and the defender are generally not simultaneous, so it is of better research value to use the dynamic game theory to build the network attack and defense model.

As a typical dynamic game, signaling game has attracted the attention of researchers for its extraordinary ability to accurately describe the critical role of intelligence information in the strategy selection of both attack and defense sides [11]. For stealthy link flooding attacks, Aydeger et al. [12] used a signaling game to perform modeling and, by solving a Bayesian Nash equilibrium, developed appropriate defense strategies and improved their effectiveness. Chen 
et al. [13] constructed a multistage signaling game model, analyzed spear-phishing attacks on networks, considered defense effectiveness and strategy cost, and proposed an optimal strategy selection algorithm. Shen et al. [14] analyzed the intrusion detection mechanism of Internet of things (IoT) with multistage signaling game and calculated the best malware detection strategy to inhibit the spread of malware.

Considering that there is only one state in multistage games, some researcher combined Markov decision process with game model to realize the state transition of the game. Considering the influence of network attack on the power grid system, Li et al. [15] analyzed the network security state assessment from the perspective of defenders and put forward the network security state assessment method with Markov game model as the core. Tan et al. [9] proposed an attack-defense game model for moving targets based on the attack surface and detection surface and built multilevel and multistate features in the process of attack and defense confrontation by using Markov robust game model. On this basis, an optimal defense strategy selection algorithm for moving targets was proposed. Sahabandu et al. [16] adopted Markov game to model Dynamic Information Flow Tracking (DIFT) and Advanced Persistent Threats (APTs) as a two-person zero-sum game model and proposed Q-learning algorithm to solve the problem of APTs attack detection. Li et al. [17] considered the optimal decisionmaking method of moving target defense and used Markov game method to calculate the optimal strategy in time and space, thus improving the value of the decision.

Multistage game models can represent network attack and defense to some extent, but discrete attack-defense processes are far from applicable to actual attack-defense confrontations. As a theoretical method to study conflict confrontations and decision-making processes in continuous time [18], differential game can model and analyze the rapid and continuous change of the network attack-defense process. Huang and Zhu [19] used a differential game framework to analyze antivirus mechanisms on complex networks and proposed a distributed antivirus algorithm to minimize the overall network cost. M. Li and S. Li [20] established a differential game model in wireless sensor networks, studied network IP defense problems, and proposed an optimal defense strategy based on limited resources. Feng et al. [21] used differential game theory to model the attack-defense interaction process of an objective function attack (OFA), introduced threat factors to describe the threats in the system operating environment, and determined the optimal operating node parameters through numerical calculations. Yang et al. [22] studied the repair problem of APT attack and used differential game to allocate available repair resources to insecure hosts, so as to reduce the potential loss of the organization. Aiming at the attack decision-making problem in honeynet, Ren and Zhang [23] considered the inherent characteristics and functional interaction between ordinary nodes and honeypots, established a differential game model, and finally obtained the optimal dynamic strategies for honeynet system and attacker.
Considering the randomness of network system states and attack-defense strategies, and their effects on the states of the game system, Orojloo and Azgomi [24] proposed to assess network security using a stochastic game model with the two phases of intrusion and interruption. Liu et al. [25] analyzed the impact of threat propagation on the security of a network system, evaluated system states through a stochastic game model, and provided enhanced defense strategies based on the Nash equilibrium. Kandoussi et al. [26] combined stochastic game theory with attack graphs to model attack-defense interactions, analyzed potential attack paths, and deployed corresponding security measures.

Although differential game models can solve continuous real-time decision-making problems for attack-defense confrontation, they do not consider the influence of uncertain factors in confrontation processes. Attack-defense strategies are inevitably affected by fluctuations in equipment performance and changes in network environments during strategy execution. Therefore, the effectiveness of strategies fluctuates. Stochastic game models describe the influence of random factors in the game process through state jumps, but current models adopt staged game methods, which provide limited guidance for the continuous real-time selection of a network defense strategy.

To deal with the inability of stochastic games at continuous real-time decision-making and the lack of random disturbances in differential games, we construct an attackdefense stochastic differential game model. By solving and analyzing the saddle point equilibrium, we design an algorithm to select an optimal defense strategy and verify the effectiveness of the model and method through simulation experiments. Our main contributions are as follows. (1) We propose a network attack-defense stochastic differential game model, consider the influence of random factors in the execution of attack-defense strategies, and change the intensity factor of random disturbance and characterize the difference in the random disturbance effects on the players. Our model is more suitable than network attack-defense models based on a classic differential game for real-world network attack and defense scenarios. (2) To describe the selection of attack-defense strategies and changes in payoffs, we construct an attackdefense decision-making control function and payoff integral function. Based on the Hamilton-Jacobi-Bellman (HJB) equation, we solve the saddle point equilibrium strategy, obtain the optimal dynamic strategy trajectory description equation, and design an algorithm for realtime selection of an optimal defense. Our model has better timeliness, relevance, and practicality than the existing methods. (3) We conduct simulation experiments. Based on the numerical analysis on the number of nodes in different security states and attack-defense optimal dynamic strategies, we provide decision-making recommendations for network defense scenarios with random disturbances (experimental code and data are available at https://github.com/kaster-hn/Network-attack-defensestochastic-differential-game-algorithm). 


\section{Attack-Defense Stochastic Differential Game Model}

2.1. Analysis of Network Security State Transition. The security states of nodes in a network change continuously due to virus invasion, penetration, and the network's own restoring function, which is similar to the spread and immune process of infectious diseases. Therefore, we can use the susceptible-infectious-recovered (SIR) dynamic model to study the transition of the network security state. We use the nodes in a network system to represent the individuals of the SIR model, expand that model, and construct a network security state transition model, namely, the normal-infected-restored-malfunctioned (NIRM) model, with four network node security states. Changes of node states are affected by the outcome of the confrontation between attack and defense strategies.

Normal state $N$ : A network node functions normally. Due to its inherent vulnerability, it may be attacked.

Infected state $I$ : A network node has been infected by the attack strategy but can still function normally; the attacker can also use this node to attack neighboring nodes.

Restored state $R$ : A network node has been effectively protected by the defender. The node possesses immunity, and the attacker fails to infect it.

Malfunctioned state $M$ : A network node cannot continue to provide services, because its quality of service deteriorates severely after attack.

The network security state transition model has five state transition paths:

$N \longrightarrow I:$ If the attacker successfully invades or penetrates a node in the normal state $N$, then the node transitions from state $N$ to state $I$, and the attacker can use it to attack neighboring nodes.

$N \longrightarrow R:$ A node in the normal state $N$ discovers its own vulnerabilities, removes them, and forms an immune function that prevents an attack.

$I \longrightarrow N$ : The defender identifies and modifies an infected node and transitions it to a normal node; however, due to different network environments and node configurations, the defender cannot identify all vulnerabilities. The attacker can use different strategies to attack again.

$I \longrightarrow R:$ The defender identifies infected nodes, removes all vulnerabilities, and acquires immunity, so the attacker cannot attack again.

$I \longrightarrow M$ : The infected node is destroyed by the attacker, resulting in a serious deterioration of its quality of service and the loss of its service function. The node can no longer be used to attack neighboring nodes.

Denote the total time of a network attack by $T$, and the total number of nodes by $Q$. Let the number of the nodes in states $N, I, R$, and $M$ at time $t$ be $x_{N}(t), x_{I}(t), x_{R}(t)$, and $x_{M}(t)$, respectively, $\forall t \in\left[t_{0}, T\right], x_{N}(t), x_{I}(t), x_{R}(t), x_{M}(t)$ $\geq 0$ and. $x_{N}(t), x_{I}(t), x_{R}(t), x_{M}(t)=Q$.
According to the above five state transition scenarios, the state transition of a node is carried out around states $N$ and $I$, because these two states can be restored and infected. The infection action is analyzed first. A node in state $I$ can only infect nodes directly connected to it. Therefore, the more nodes connected to state $I$, the faster the growth rate of infected nodes. Assuming that the nodes in the network are distributed with density $\sigma$, the number of nodes connected to a node is $\sigma \pi r^{2}$, where $r$ is the network distance between two nodes. When $r=1$, two nodes are directly connected. Therefore, the number of nodes directly connected to an infected node in state $I$ is $\sigma \pi$. At time $t$, the proportion of normal nodes is $x_{N}(t) / Q$, and hence, the number of normal nodes adjacent to an infected node is $\sigma \pi x_{I}(t) x_{N}(t) / Q$. Assume that the infection rate is $v_{a}$, and this is related to the proportion of infected nodes in the network. Let $v_{a}=\alpha x_{I}(t) / Q$, where $\alpha$ is an infection rate coefficient. Then, in an attack-defense confrontation, if the defense strategy fails, the number of nodes transitioning from state $N$ to state $I$ is $v_{a} \sigma \pi x_{I}(t) x_{N}(t) / Q$, and the number transitioning from state $I$ to state $M$ is $v_{a} x_{I}(t)$. The nodes are restored through the defense strategy, which, if successful, can have two results: entering the normal state and entering the restored state. The specific state depends on the network structure and the strategies adopted by the attacker and defender. Assume that the probability of entering the restored state is a constant $\mu$, and the restoring rate $v_{d}$ is related to the ratio of normal to restored nodes in the network. Let $v_{d}=\beta\left(x_{N}(t)+x_{I}(t)\right) / Q$, where $\beta$ is a restoring rate coefficient. Then, in the attack-defense confrontation, if the defense strategy is successful, the numbers of nodes transitioning from state $I$ to $N, I$ to $R$, and $N$ to $R$ are $(1-\mu) v_{d} x_{I}(t), \mu v_{d} x_{I}(t)$, and $\mu v_{d} x_{N}(t)$, respectively.

Defenders can be divided into those of high-, medium-, and low-intensity types, denoted by $D_{H}, D_{M}$, and $D_{L}$, respectively, with corresponding defense strategy strengths of $\overrightarrow{s_{D}^{H}}, \overline{s_{D}^{M}}, \overline{s_{D}^{L}} \in[0,1]$. The defender adopts a hybrid strategy, selecting its defense strategy at time $t$ according to the probability vector $P_{D}(t)=\left(p_{D}^{H}(t), p_{D}^{M}(t), p_{D}^{L}(t)\right)$. The expected utilities of high-, medium-, and low-intensity defense strategies are $d_{h}(t)=p_{D}^{H}(t) \overline{s_{D}^{H}}, \quad d_{m}(t)=p_{D}^{M}(t) s_{D}^{M}$, and $d_{l}(t)=p_{D}^{L}(t) \overline{s_{D}^{L}}$, respectively. So, at time $t$, the overall expected utility of the defender is $d(t)=d_{h}(t)+d_{m}(t)+d_{l}(t)$. Attackers are similarly divided into those of high-, medium-, and low-intensity types, denoted by $A_{H}, A_{M}$, and $A_{L}$, respectively, with corresponding attack strategy strengths denoted by $\overline{s_{A}^{H}}, \overline{s_{A}^{M}}, \overline{s_{A}^{L}} \in[0,1]$. At time $t$, the attacker adopts a hybrid strategy, $P_{A}(t)=\left(p_{A}^{H}(t), p_{A}^{M}(t), p_{A}^{L}(t)\right)$. The expected utilities of high-, medium-, and low-intensity attack strategies are $a_{h}(t)=p_{A}^{H}(t) \overline{s_{A}^{H}}, \quad a_{m}(t)=p_{A}^{M}(t) \overline{s_{A}^{M}}$, and $a_{l}(t)=p_{A}^{L}(t) \overline{s_{A}^{L}}$, respectively. So, the overall expected utility of the attacker at time $t$ is $a(t)=a_{h}(t)+a_{m}(t)+a_{l}(t)$.

However, in a real-world network attack-defense confrontation, the network environment changes constantly and randomly. The defender always has certain information errors in its observation of the network environment. At the same time, in the execution of defense strategies, the basic network quality of service, performance of network nodes, and defense equipment are dynamic and may vary randomly 
within a certain range; i.e., they are not static. Therefore, an actual defense utility is a random variable. Suppose that, at time $t$, the actual defense utility of defender type $i$ is $d_{i}(t)=p_{D}^{i}(t) \overline{s_{D}^{i}}+\delta_{D} d \omega_{D}^{i}(t), i \in\{H, M, L\}$, where $\delta_{D}$ is the factor of random disturbance intensity, $\omega_{D}^{i}(t)$ reflects onedimensional Brownian motion and is distributed as $N\left(0,\left(\rho_{D} s_{D}^{i}\right)^{2} t\right), d \omega_{D}^{i}(t)$ represents random disturbance and is distributed as $N\left(0,\left(\rho_{D} s_{D}^{i}\right)^{2} \Delta t\right)$, and $\rho_{D}$ is the coefficient of variation. The random disturbances received by the defender mainly come from the fluctuation of the basic network environment and equipment performance. The intensity of random disturbance received by different types of defenders is basically the same and is denoted by $\delta_{D}$. The high-intensity strategy is more complex, requires more resources in execution, is affected by other processes in the system, and has a higher execution cost. Therefore, its actual utility will be more instable, i.e., with greater variance. Assume that the variance and defense strength are positively correlated as $\left(\rho_{D} s_{D}^{i}\right)^{2} \Delta t$. Then, the actual defense utility of the defender at time $t$ is $d_{\text {act }}(t)=d_{H}(t)+d_{M}(t)+d_{L}(t)$.

Similarly, the actual attack utility is affected by uncertain factors. In particular, due to the asymmetry of information in a network attack-defense confrontation, attack information tends to be more concealed and difficult to observe. The grasp of game information such as attack strategies and attack revenue calculations is incomplete and uncertain. The calculation of actual attack utility has higher uncertainty; i.e., the attacker receives greater random disturbance intensity. Suppose that, at time $t$, the actual attack utilities by various types of attackers are $a_{i}(t)=p_{A}^{i}(t) \overline{s_{A}^{i}}+\delta_{A} d \omega_{A}^{i}(t)$, $i \in\{H, M, L\}$, where $\delta_{A}$ is the random disturbance intensity factor, $\omega_{A}^{i}(t)$ is one-dimensional Brownian motion distributed as $N\left(0,\left(\rho_{A} s_{A}^{i}\right)^{2} t\right), d \omega_{A}^{i}(t)$ is distributed as $N\left(0,\left(\rho_{A} s_{A}^{i}\right)^{2} \Delta t\right)$, and $\rho_{A}$ is the coefficient of variation. The attack strategy is subjected to the same intensity of random disturbance, $\delta_{A}$, during execution. From the above analysis, $\delta_{A}>\delta_{D}$. The actual attack utility of the attacker at time $t$ is $a_{\text {act }}(t)=a_{H}(t)+a_{M}(t)+a_{L}(t)$.

The result of the attack-defense confrontation determines the state transition of the nodes, and the difference between the actual utilities of the attack-defense hybrid strategies, $\tau(t)=a_{\mathrm{act}}(t)-d_{\mathrm{act}}(t)$, is used to represent the confrontation result. Note that $|\tau(t)| \in[0,1]$. The attack is successful if $\tau(t) \geq 0$, and the defense is successful if $\tau(t)<0$. Using attack-defense results, $\tau(t)$, to analyze the state transition paths, we can obtain the parameters $\tau_{N I}, \tau_{N R}, \tau_{I N}$, $\tau_{I R}$, and $\tau_{I M}$ to describe the likelihoods of state transitions $N \longrightarrow I, N \longrightarrow R, I \longrightarrow N, I \longrightarrow R$, and $I \longrightarrow M$, respectively:

$$
\begin{gathered}
\tau_{N I}=\tau_{I M}= \begin{cases}0, & \tau(t)<0 \\
\tau(t), & \tau(t) \geq 0\end{cases} \\
\tau_{N R}=\tau_{I N}=\tau_{I R}= \begin{cases}|\tau(t)|, & \tau(t)<0 \\
0, & \tau(t) \geq 0\end{cases}
\end{gathered}
$$

Based on the above analysis, the node state transition equations are obtained as

$$
\left\{\begin{array}{l}
\dot{x}_{N}=\frac{-\tau_{N I}(t) v_{a} \sigma \pi x_{I}(t) x_{N}(t)}{Q-\mu \tau_{N R}(t) v_{d} x_{N}(t)+(1-\mu) \tau_{I N}(t) v_{d} x_{I}(t)} \\
\dot{x}_{I}=\frac{\tau_{N I}(t) v_{a} \sigma \pi x_{I}(t) x_{N}(t)}{Q-\tau_{I M}(t) v_{a} x_{I}(t)-(1-\mu) \tau_{I N}(t) v_{d} x_{I}(t)-\mu \tau_{I R} v_{d} x_{I}(t)} \\
\dot{x}_{R}=\mu \tau_{N R}(t) v_{d} x_{N}(t)+\mu \tau_{I R} v_{d} x_{I}(t) \\
\dot{x}_{M}=\tau_{I M}(t) v_{a} x_{I}(t) \\
\forall t \in\left[t_{0}, T\right] \\
x_{N}(t)+x_{I}(t)+x_{R}(t)+x_{M}(t)=Q
\end{array}\right.
$$

\subsection{Definition of Attack-Defense Stochastic Differential Game Model}

Definition 1. The network attack-defense stochastic differential game model $A D S D G$ can be expressed as a nine-tuple, $A D S D G=(\mathfrak{R}, \mathfrak{J}, P, t, x, \delta, P, \theta, U)$.
(1) $\mathfrak{R}=\left(\mathfrak{R}_{D}, \mathfrak{R}_{A}\right)$ is a set of game players, where $\mathfrak{R}_{D}$ and $\mathfrak{R}_{A}$ represent defenders and attackers, respectively.

(2) $\mathfrak{I}=\left(\mathfrak{I}_{D}, \mathfrak{I}_{A}\right)$ is the type space of the defender and attacker. It is the private information of the players. $\mathfrak{R}_{D}$ $=\left\{A_{i} \mid i=1,2, \ldots, m\right\}$ and $\mathfrak{R}_{A}=\left\{A_{j} \mid j=1,2, \ldots, n\right\}$. 
(3) $\wp=(D G, A G)$ is the action space of players. $D G=$ $\left(\zeta_{1}, \zeta_{2}, \ldots, \zeta_{q}\right)$ and $A G=\left(\xi_{1}, \xi_{2}, \ldots, \xi_{k}\right)$ are the action sets of the defender and attacker, respectively. $g, k \geq 1$.

(4) $t$ is the moment of the attack-defense game, $t \in\left[t_{0}, T\right]$.

(5) $x(t)=\left\{\left(x_{N}(t), x_{I}(t), x_{R}(t), x_{M}(t)\right) \mid x_{N}(t)+x_{I}(t)+\right.$ $\left.x_{R}(t)+x_{M}(t)=Q\right\}$ is the state variable of nodes in the network. $x_{N}(t), x_{I}(t), x_{R}(t), x_{M}(t)$ represent the number of nodes in the normal, infected, restored, and malfunctioned states, respectively, at time $t$. $Q$ is the total number of nodes.

(6) $\delta=\left(\delta_{D}, \delta_{A}\right)$ is the random disturbance intensity factor, which indicates how much the random disturbance affects the actual utility of the strategy. Random disturbances received by the defender mainly come from the influence of the basic network environment and equipment performance. The attacker is not only affected by the above random disturbances. The asymmetry of attack and defense information increases the difficulty of calculating the actual attack utility, which leads to a higher random disturbance intensity, i.e., $\delta_{A}>\delta_{D}$.

(7) $P=\left(P_{D}(t), P_{A}(t)\right)$ is the dynamic strategy of the attacker and defender at time $t$. $P_{D}(t)=\left\{p_{D}^{i}(t) \mid 1 \leq i \leq m\right\}$ represents the hybrid strategy of the defender at time $t$, where $p_{D}^{i}(t)$ is the probability that the defender chooses defense strategy type $i, \quad$ and $\quad \sum_{i=1}^{m} p_{D}^{i}(t)=1$. Similarly, $P_{A}(t)=\left\{p_{A}^{j}(t) \mid 1 \leq j \leq n\right\} \quad$ represents the hybrid strategy of the attacker at time $t, p_{A}^{j}(t)$ is the probability of the attacker choosing attack strategy type $i$, and $\sum_{j=1}^{n} p_{A}^{j}(t)=1$.

(8) $\theta=\left\{\theta_{N}, \theta_{I}, \theta_{R}, \theta_{M}\right\}$ is the state transition function, where $\theta_{N}=\mathrm{d} x_{N}(t) / \mathrm{d} t=\dot{x}_{N}, \quad \theta_{I}=\mathrm{d} x_{I}(t) / \mathrm{d} t=\dot{x}_{I}$, $\theta_{R}=\mathrm{d} x_{R}(t) / \mathrm{d} t=\dot{x}_{R}, \theta_{M}=\mathrm{d} x_{M}(t) / \mathrm{d} t=\dot{x}_{M}$.

(9) $L=\left(L_{D}, L_{A}\right)$ is the set of payoff functions of the defender and attacker. In the stochastic differential game model, a payoff function is
$L=\int_{t_{0}}^{T} g\left(t, x(t), P_{A}(t), P_{D}(t)\right) \mathrm{d} t+q(x(t)), \quad$ where $g:[0, T] \times \mathbb{R}^{4} \times P_{A} \times P_{D} \mapsto \mathbb{R}$ represents the instantaneous payoffs of the players at time $t$, and $q: x(T) \mapsto \mathbb{R}$ represents the final payoff at the end of the game.

\section{Equilibrium Solution and Selection of Optimal Defense Strategy}

3.1. Equilibrium Solution of Attack-Defense Stochastic Differential Game. To facilitate the solution of the attackdefense stochastic differential game, the game's equilibrium solution is divided into two parts: quantifying payoffs and solving the saddle point equilibrium strategy. Payoff quantification is the basis for solving the saddle point equilibrium strategy, whose method is related to the result of the equilibrium solution and affects the selection of the optimal defense strategy.

3.1.1. Payoff Quantification. Based on the analysis in Section 2.1 and the definition of the attack-defense stochastic differential game model in Section 2.2, the following method of payoff quantification is proposed. When a node experiences a state transition, the attacker and defender are affected accordingly. The reward coefficient of a node transitioning from state $N$ to state $I$ is denoted by $r_{1}$, representing the damage to neighboring nodes after the node is infected. The reward coefficient of a node transitioning from state $N$ or $I$ to state $R$ is denoted by $r_{2}$, representing immunity of the node after being restored, which may reduce the expected loss. The reward coefficient of the node from state $I$ to state $M$ is denoted by $r_{3}$, representing the loss of the node's service function. The reward coefficient of the node from state $I$ to state $N$ is denoted by $r_{4}$. The statistical average approach is used to define the reward coefficients $r_{1}, r_{2}, r_{3}, r_{4} \in[0,10]$. In addition, when a node transitions to state $M$, a new normal node needs to be established, with terminal cost $c_{M}^{T} x_{M}(T)$, where $c_{M}^{T} \geq 0$.

Therefore, the rewards for the defender and attacker, respectively, at time $t$ are

$$
\begin{aligned}
& r_{D}=\frac{r_{2}\left(\mu \tau_{N R}(t) v_{d} x_{N}(t)+\mu \tau_{I R} v_{d} x_{I}(t)\right)+r_{4}(1-\mu) \tau_{I N}(t) v_{d} x_{I}(t)-r_{3} \tau_{I M}(t) v_{a} x_{I}(t)-r_{1} \tau_{N I}(t) v_{a} \sigma \pi x_{I}(t) x_{N}(t)}{Q}, \\
& r_{A}=\frac{r_{1} \tau_{N I}(t) v_{a} \sigma \pi x_{I}(t) x_{N}(t)}{Q+r_{3} \tau_{I M}(t) v_{a} x_{I}(t)-r_{2}\left(\mu \tau_{N R}(t) v_{d} x_{N}(t)+\mu \tau_{I R} v_{d} x_{I}(t)\right)-r_{4}(1-\mu) \tau_{I N}(t) v_{d} x_{I}(t)} .
\end{aligned}
$$

The attacker and defender will incur costs associated with strategy execution. These are related to utilities. The higher a strategy's utility, the greater the cost. Denote the cost coefficients of high-, medium-, and low-intensity defense strategies by $c_{D}^{H}, c_{D}^{M}$, and $c_{D}^{L}$, respectively, and those of high-, medium-, and low-intensity attack strategies by $c_{A}^{H}$, $c_{A}^{M}$, and $c_{A}^{L}$, respectively, where $c_{i}^{H}>c_{i}^{M}>c_{i}^{L}, i \in\{D, A\}$. By referring to [27], the strategy costs of defense and attack, respectively, at time $t$ are established as

$$
\begin{gathered}
c_{D}=\frac{1}{2}\left(d_{H}^{2} c_{D}^{H}+d_{M}^{2} c_{D}^{M}+d_{L}^{2} c_{D}^{L}\right)\left(x_{N}(t)+x_{R}(t)\right), \\
c_{A}=\frac{1}{2}\left(a_{H}^{2} c_{A}^{H}+a_{M}^{2} c_{A}^{M}+a_{L}^{2} c_{A}^{L}\right) x_{I}(t) .
\end{gathered}
$$


Through the above analysis and comprehensive consideration of the costs and rewards of attack-defense strategies, the payoff functions of the attacker and defender can be obtained as

$$
\left\{\begin{array}{l}
L_{D}\left(P_{A}(t), P_{D}(t)\right)=\int_{t_{0}}^{T} g_{D}\left(t, x(t), P_{A}(t), P_{D}\right) \mathrm{d} t+q_{D}(x(T)) \\
=\int_{t_{0}}^{T}\left[\frac{r_{2}\left(\mu \tau_{N R}(t) v_{d} x_{N}(t)+\mu \tau_{I R} v_{d} x_{I}(t)\right)+r_{4}(1-\mu) \tau_{I N}(t) v_{d} x_{I}(t)-r_{3} \tau_{I M}(t) v_{a} x_{I}(t)-r_{1} \tau_{N I}(t) v_{a} \sigma \pi x_{I}(t) x_{N}(t)}{Q-1 / 2\left(d_{H}^{2} c_{D}^{H}+d_{M}^{2} c_{D}^{M}+d_{L}^{2} c_{D}^{L}\right)\left(x_{N}(t)+x_{R}(t)\right.}\right]-c_{M}^{T} x_{M}(T) \\
L_{A}\left(P_{A}(t), P_{D}(t)\right)=\int_{t_{0}}^{T} g_{A}\left(t, x(t), P_{A}(t), P_{D}(t)\right) d t+q_{A}(x(T)) \\
=\int_{t_{0}}^{T}\left[\frac{r_{1} \tau_{N I}(t) v_{a} \sigma \pi x_{I}(t) x_{N}(t)}{Q+r_{3} \tau_{I M}(t) v_{a} x_{I}(t)-r_{2}\left(\mu \tau_{N R}(t) v_{d} x_{N}(t)+\mu \tau_{I R} v_{d} x_{I}(t)\right)-r_{4}(1-\mu) \tau_{I N}(t) v_{d} x_{I}(t)-1 / 2\left(a_{H}^{2} c_{A}^{H}+a_{M}^{2} c_{A}^{M}+a_{L}^{2} c_{A}^{L}\right) x_{I}(t)}\right] \mathrm{d} t \\
d_{H}=p_{D}^{H} \overline{s_{D}^{H}}+\delta_{D} d W_{D}^{H}, \\
d_{M}=p_{D}^{M} \overline{s_{D}^{M}}+\delta_{D} d W_{D}^{M} \\
d_{L}=p_{D}^{L} \overline{s_{D}^{L}}+\delta_{D} d W_{D}^{L}, \\
a_{H}=p_{A}^{H} \overline{s_{A}^{H}}+\delta_{A} d W_{A}^{H}, \\
a_{M}=p_{A}^{M} \overline{s_{A}^{M}}+\delta_{A} d W_{A}^{M} \\
a_{L}=p_{A}^{L} \overline{s_{A}^{L}}+\delta_{A} d W_{A}^{L} .
\end{array}\right.
$$

3.1.2. Solution of Saddle Point Equilibrium Strategy. For a network attack-defense stochastic differential game model $A D S D G$, both the attacker and defender want to maximize their payoff by choosing optimal strategies. Let $\left(P_{D}(t)^{*}, P_{A}(t)^{*}\right)$ represent the optimal dynamic strategies of the defender and attacker.
Definition 2. If the strategy set $\left(P_{D}(t)^{*}, P_{A}(t)^{*}\right)$ constitutes a Nash equilibrium solution of the model $A D S D G$, then the following equations exist:

$$
\left\{\begin{array}{l}
P_{D}(t)^{*}=\max _{P_{D}(t)}\left[\int_{t_{0}}^{T} g_{D}\left(t, x(t)^{*}, P_{A}(t)^{*}, P_{D}(t)\right) \mathrm{d} t+q_{D}\left(x(T)^{*}\right)\right], \\
P_{A}(t)^{*}=\max _{P_{A}(t)}\left[\int_{t_{0}}^{T} g_{A}\left(t, x(t)^{*}, P_{A}(t), P_{D}(t)^{*}\right) \mathrm{d} t+q_{A}\left(x(T)^{*}\right)\right], \\
\dot{x}(t)^{*}=\theta\left(t, x(t)^{*}, P_{A}(t)^{*}, P_{D}(t)^{*}\right) .
\end{array}\right.
$$

$\left(P_{A}^{*}(t), P_{D}^{*}(t)\right)$ is referred to as the saddle equilibrium strategy in the attack-defense stochastic differential game, or the saddle point strategy in short.
Theorem 1. For the attack-defense stochastic differential game model ADSDG, if there is a costate function $\lambda_{i}(t):\left[t_{0}, T\right] \times \mathbb{R}^{4} \longrightarrow \mathbb{R}, i \in\{D, A\}$, then a saddle point 
$\left(P_{D}(t)^{*}, P_{A}(t)^{*}\right)$ strategy exists if the following conditions are met, where $P_{-i}^{*}(t)$ denotes the collection of controls of all players except player $i$.

$$
\left\{\begin{array}{l}
\frac{\mathrm{d}}{\mathrm{d} t} x^{*}(t)=\theta\left(t, x^{*}(t), P_{D}^{*}(t), P_{A}^{*}(t)\right), \\
x^{*}\left(t_{0}\right)=x\left(t_{0}\right), \\
P_{i}^{*}(t)=\underset{P_{i}(t)}{\arg \max }\left[\theta\left(t, x^{*}(t), P_{i}(t), P_{-i}^{*}(t)\right) \lambda_{i}(t)+g_{i}\left(t, x^{*}(t), P_{i}(t), P_{-i}^{*}(t)\right)\right], \\
\frac{\mathrm{d}}{\mathrm{d} t} \lambda_{i}(t)=-\frac{\partial}{\partial x^{*}}\left[\theta\left(t, x^{*}(t), P_{i}^{*}(t), P_{-i}^{*}(t)\right) \lambda_{i}(t)+g_{i}\left(t, x^{*}(t), P_{i}^{*}(t), P_{-i}^{*}(t)\right)\right], \\
\lambda_{i}(T)=\frac{\partial q_{i}\left(x^{*}(T)\right)}{\partial x^{*}} .
\end{array}\right.
$$

Proof. In Theorem 1, when the attacker chooses the optimal strategy $P_{A}^{*}(t)$, the defender forms an optimal control problem. According to Pontryagin's maximum principle, the existence of the function $\lambda_{D}(t)$ can be proved, and the existence of the function $\lambda_{A}(t)$ can similarly be proved. Therefore, Theorem 1 is proved: there is a saddle point strategy in the attack-defense stochastic differential game ADSDG.

The solution process for the saddle point strategy is as follows. The Hamiltonian function of the defender is constructed as

$$
\begin{aligned}
H_{D}\left(t, \lambda_{D}(t), x(t), P_{A}(t), P_{D}(t)\right)= & g_{D}\left(t, x(t), P_{D}(t), P_{A}(t)\right)+\sum_{i \in\{N, I, R, M\}} \frac{\partial \lambda_{D}}{\partial x_{i}} \theta_{i}\left(t, x(t), P_{D}(t), P_{A}(t)\right) \\
= & \tau_{N R}(t)\left[\left(r_{2}-\lambda_{D}^{x_{N}}+\lambda_{D}^{x_{R}}\right) \mu v_{d} x_{N}(t)\right]+\tau_{I R}(t)\left[\left(r_{2}-\lambda_{D}^{x_{I}}+\lambda_{D}^{x_{R}}\right) \mu v_{d} x_{I}(t)\right] \\
& +\tau_{I N}(t)\left[\left(r_{4}+\lambda_{D}^{x_{N}}-\lambda_{D}^{x_{I}}\right)(1-\mu) v_{d} x_{I}(t)\right]+\tau_{N I}(t)\left[\frac{\left(-r_{1}-\lambda_{D}^{x_{N}}+\lambda_{D}^{x_{I}}\right) v_{a} \sigma \pi x_{I}(t) x_{N}(t)}{Q}\right] \\
& +\tau_{I M}(t)\left[\left(-r_{3}-\lambda_{D}^{x_{I}}+\lambda_{D}^{x_{M}}\right) v_{a} x_{I}(t)\right]-\frac{1}{2}\left(d_{H}^{2} c_{D}^{H}+d_{M}^{2} c_{D}^{M}+d_{L}^{2} c_{D}^{L}\right)\left(x_{N}(t)+x_{R}(t)\right) .
\end{aligned}
$$


Then, the costate function $\lambda_{D}(t)$ is calculated.

$$
\lambda_{D}(t)=\left(\lambda_{D}^{x_{i}}(t)\right)^{T}=\left(\lambda_{D}^{x_{N}}(t), \lambda_{D}^{x_{I}}(t), \lambda_{D}^{x_{R}}(t), \lambda_{D}^{x_{M}}(t)\right)^{T} .
$$

$$
\begin{aligned}
& \left\{\begin{array}{l}
\frac{\mathrm{d}}{\mathrm{d} t} \lambda_{D}^{x_{N}}(t)=-\frac{\partial}{\partial x_{N}(t)} H_{D}\left(t, \lambda(t), x(t), P_{A}(t), P_{D}(t)\right) \\
=-\frac{\tau_{N R}(t) \mu \beta\left(r_{2}-\lambda_{D}^{x_{N}}+\lambda_{D}^{x_{R}}\right)\left(x_{R}(t)+2 x_{N}(t)\right)}{Q}-\frac{\tau_{I R}(t) \mu \beta\left(r_{2}-\lambda_{D}^{x_{I}}+\lambda_{D}^{x_{R}}\right) x_{I}(t)}{Q} \\
-\frac{\tau_{I N}(t)(1-\mu) \beta\left(r_{4}+\lambda_{D}^{x_{N}}-\lambda_{D}^{x_{I}}\right) x_{I}(t)}{Q}-\frac{\tau_{N I}(t) \alpha\left(-r_{1}-\lambda_{D}^{x_{N}}+\lambda_{D}^{x_{I}}\right) \sigma \pi x_{I}^{2}(t)}{Q^{2}}+\frac{1}{2}\left(d_{H}^{2} c_{D}^{H}+d_{M}^{2} c_{D}^{M}+d_{L}^{2} c_{D}^{L}\right) \\
\lambda_{D}^{x_{N}}(t)=0
\end{array}\right. \\
& \left\{\begin{array}{l}
\frac{\mathrm{d}}{\mathrm{d} t} \lambda_{D}^{x_{I}}(t)=-\frac{\partial}{\partial x_{I}(t)} H_{D}\left(t, \lambda(t), x(t), P_{A}(t), P_{D}(t)\right) \\
=-\frac{\tau_{I R}(t) \mu \beta\left(r_{2}-\lambda_{D}^{x_{I}}+\lambda_{D}^{x_{R}}\right)\left(x_{N}(t)+x_{R}(t)\right)}{Q}-\frac{\tau_{I N}(t)(1-\mu) \beta\left(r_{4}+\lambda_{D}^{x_{N}}-\lambda_{D}^{x_{I}}\right)\left(x_{N}(t)+x_{R}(t)\right)}{Q} \\
\quad-\frac{2 \tau_{N I}(t) \alpha\left(-r_{1}-\lambda_{D}^{x_{N}}+\lambda_{D}^{x_{I}}\right) \sigma \pi x_{I}(t) x_{N}(t)}{Q^{2}}-\frac{2 \tau_{I M}(t) \alpha\left(-r_{3}-\lambda_{D}^{x_{I}}+\lambda_{D}^{x_{M}}\right) x_{I}(t)}{Q} \\
\lambda_{D}^{x_{I}}(T)=0
\end{array}\right. \\
& \left\{\begin{array}{l}
\frac{\mathrm{d}}{\mathrm{d} t} \lambda_{D}^{x_{R}}(t)=-\frac{\partial}{\partial x_{R}(t)} H_{D}\left(t, \lambda(t), x, P_{A}(t), P_{D}(t)\right) \\
=-\frac{\tau_{N R}(t) \mu \beta\left(r_{2}-x_{N}(t)+\lambda_{D}^{x_{R}}\right) x_{N}(t)}{Q}-\frac{\tau_{I R}(t) \mu \beta\left(r_{2}-\lambda_{D}^{x_{I}}+\lambda_{D}^{x_{R}}\right) x_{I}(t)}{Q} \\
-\frac{\tau_{I N}(t)(1-\mu) \beta\left(r_{4}+x_{N}(t)-\lambda_{D}^{x_{I}}\right) x_{I}(t)}{Q+(1 / 2)\left(d_{H}^{2} c_{D}^{H}+d_{M}^{2} c_{D}^{M}+d_{L}^{2} c_{D}^{L}\right)}, \\
\lambda_{D}^{x_{R}}(T)=0,
\end{array}\right. \\
& \left\{\begin{array}{l}
\frac{\mathrm{d}}{\mathrm{d} t} \lambda_{x_{M}}(t)=-\frac{\partial}{\partial x_{M}(t)} H_{D}\left(t, \lambda(t), x(t), P_{A}(t), P_{D}(t)\right)=0, \\
\lambda_{x_{M}}(T)=-c_{M}^{T} .
\end{array}\right.
\end{aligned}
$$

Let $\partial H_{D}^{*} / \partial p_{D}^{i}(t)=0$. Then, $p_{D}^{i}(t)^{*}$ is obtained as

$$
p_{D}^{i}(t)^{*}=\left\{\begin{array}{l}
\frac{\left(r_{1}+\lambda_{D}^{x_{N}}-\lambda_{D}^{x_{I}}\right) \sigma \pi v_{a} x_{I}(t) x_{N}(t) / Q+\left(r_{3}+\lambda_{D}^{x_{I}}-\lambda_{D}^{x_{M}}\right) v_{a} x_{I}(t)-c_{D}^{i}\left(x_{N}(t)+x_{R}(t)\right) \delta_{D}^{i} \mathrm{~d} W_{D}^{i}}{\overline{s_{D}^{i}} c_{D}^{i}\left(x_{N}(t)+x_{R}(t)\right)}, \quad \tau(t) \geq 0, \\
\frac{\left(r_{2}-\lambda_{D}^{x_{N}}+\lambda_{D}^{x_{R}}\right) \mu v_{d} x_{N}(t)+\left(r_{2}-\lambda_{D}^{x_{I}}+\lambda_{D}^{x_{R}}\right) \mu v_{d} x_{I}(t)+\left(r_{4}-\lambda_{D}^{x_{I}}+\lambda_{D}^{x_{N}}\right)(1-\mu) v_{d} x_{I}(t)-c_{D}^{i}\left(x_{N}(t)+x_{R}(t)\right) \delta_{D}^{i} \mathrm{~d} W_{D}^{i}}{\overline{s_{D}^{i}} c_{D}^{i}\left(x_{N}(t)+x_{R}(t)\right)} \quad \tau(t)<0 .
\end{array}\right.
$$


$\lambda_{A}(t)$ can be similarly obtained. Let $\partial H_{A}^{*} / \partial p_{A}^{i}(t)=0$.

Then, $p_{A}^{i}(t)^{*}$ is obtained as

$$
p_{A}^{i}(t)^{*}=\left\{\begin{array}{l}
\frac{\left(r_{1}-\lambda_{A}^{x_{N}}+\lambda_{A}^{x_{I}}\right) \sigma \pi v_{a} x_{I}(t) x_{N}(t) / Q+\left(r_{3}-\lambda_{A}^{x_{I}}+\lambda_{A}^{x_{M}}\right) v_{a} x_{I}(t)-c_{A}^{i} x_{I}(t) \delta_{A}^{i} \mathrm{~d} W_{A}^{i}}{\overline{s_{A}} c_{A}^{i} x_{I}(t)}, \quad \tau(t) \geq 0, \\
\frac{\left(r_{2}+\lambda_{A}^{x_{N}}-\lambda_{A}^{x_{R}}\right) \mu v_{d} x_{N}(t)+\left(r_{2}+\lambda_{A}^{x_{I}}-\lambda_{A}^{x_{R}}\right) \mu v_{d} x_{I}(t)+\left(r_{4}+\lambda_{A}^{x_{I}}-\lambda_{A}^{x_{N}}\right)(1-\mu) v_{d} x_{I}(t)-c_{A}^{i} x_{I}(t) \delta_{D}^{i} \mathrm{~d} W_{D}^{i}}{\overline{s_{A}^{i}} c_{A}^{i} x_{I}(t)}, \quad \tau(t)<0 .
\end{array}\right.
$$

In equations (13) and (14), $i \in\{H, M, L\}$, and $\tau(t)=a_{\text {act }}(t)-d_{\text {act }}(t)$ represents the result of the attackdefense confrontation. $p_{D}^{i}(t)^{*}$ and $p_{A}^{i}(t)^{*}$ have different results according to $\tau(t)$, indicating that the optimal strategies of the two sides are interdependent.

3.2. Algorithm to Select Optimal Defense Strategy. Algorithm to select optimal defense strategy in attack-defense differential game in Algorithm 1.

3.3. Comparative Analysis of Results. From the aspects of game type, player type, real-time performance of decisionmaking, random disturbance, equilibrium solution, and specific application, we compare and analyze the results of this paper and related results, as shown in Table 1.

In a network attack-defense confrontation, due to differences between the attacker and defender in terms of technology, ability, and tendency of strategy choice, it is more realistic to divide players into different types in the analysis. We divide both the attacker and defender into high, medium, and low types. The number of types of players can be extended to $n$, so the model can be extended to more application scenarios. However, the literatures lack a clear classification of player types and only elaborate on their action spaces. Real-time performance of decision-making refers to the effective time of one decision-making, and this has a great impact on the effectiveness of decision-making. In a staged game, the execution of a decision lasts for one stage. This way of decision-making has poor real-time performance and prevents timely responses to environment changes. By making decisions in continuous time, the stochastic differential game ensures that decision-making can adapt to changes in the network environment and has good real-time performance. Random disturbance considers the influence of uncertain factors during the attack-defense process. In an actual attack-defense confrontation, the network environment and structure are dynamically changing, and the performance of equipment varies within a certain range, which will disturb the strategy execution. In addition, the attacker and defender hide their information, making the game information incomplete for both parties. These uncertainties are unavoidable. The proposed stochastic differential game model introduces random disturbances in the strategy execution process, making the game model reflect the actual situation more realistically.
Equilibrium solution refers to whether the process of solving game equilibrium is detailed. The equilibrium solution of a stochastic differential game changes in real time, and the solution process is complex. This paper elaborates upon the detailed solving process, which can improve the practical application capability of the model.

\section{Simulation Experiment and Analysis}

4.1. Description of Experimental Environment. The simulation experiment verifies the effectiveness of the proposed network attack-defense stochastic differential game model and optimal defense strategy selection method. The Scalable Simulation Framework (SSFNet) [28-30] simulation tool is used, and parameters are set to simulate network attack and defense scenarios. The number of nodes in the simulation experiment is set to $Q=1000$. Referring to [31] and MIT's attack-defense classification [32], the attack-defense action information is given in Tables 2 and 3 .

4.2. Experimental Analysis. MATLAB 2018a is used to conduct numerical experiments. The transitions of the numbers of nodes with different security states in the network are recorded. The optimal dynamic strategies for the attacker and defender under different random disturbance intensities are calculated. On this basis, the influence of random disturbance and dynamic strategy on the transitions of node states is analyzed.

The relevant parameters in the experiment are initialized as follows. The node density is $\sigma=10$. Based on the data in Tables 2 and 3, the attacker's high-, medium-, and lowstrategy strengths are $\overline{s_{A}^{H}}=0.82, \overline{s_{A}^{M}}=0.45, \overline{s_{A}^{L}}=0.3$, respectively, and the defender's high-, medium-, and lowstrategy strengths are $\overline{s_{D}^{H}}=0.76, \overline{s_{D}^{M}}=0.5, \overline{s_{D}^{L}}=0.267$, respectively. The strategy cost factors of the attacker are $c_{A}^{H}=4.4, c_{A}^{M}=2.4, c_{A}^{L}=1.5$. The strategy cost factors of the defender are $c_{D}^{H}=4.1, c_{D}^{M}=2.7, c_{D}^{L}=1.3$. The reward coefficients for node state transition are $r_{1}=4, r_{2}=7, r_{3}=10, r_{4}=4$. The infection rate coefficient is $\alpha=0.7$. The restoring rate coefficient is $\beta=0.7$. The restored state transition probability is $\mu=0.8$. The coefficients of variation are $\rho_{A}=\rho_{D}=2$. The total number of nodes is $Q=1000$. The number of initial infected nodes is $x_{I}(0)=10$. For initial dynamic strategies, $p_{D}^{H}(t)=1$ and $p_{A}^{H}(t)=1$, indicating that the attacker and defender adopt the highest-intensity strategies at the beginning of the game. 
Input: Attack-defense stochastic differential game model ADSDG

Output: Optimal defense strategy $p_{D}^{*}(t)$

BEGIN

Initialize $A D S D G=(\Re, \mathfrak{I}, \wp, t, x, \delta, P, \theta, L)$

/ $*$ Initialize the stochastic differential game model $*$ |

Initialize $p_{D}^{H}(t)=1$ and $p_{A}^{H}(t)=1$

$/ *$ For the given initial attack-defense strategies, all adopt high-intensity strategies $* /$

Calculate, $\tau_{N I}, \tau_{N R}, \tau_{I N}, \tau_{I R}$, and $\tau_{I M}$

$/ *$ Calculate the result of attack-defense confrontation according to the initial strategy*I

FOR $t=0$ to $T$

Substitute $\tau_{N I}, \tau_{N R}, \tau_{I N}, \tau_{I R}$, and $\tau_{I M}$ in equations (2) and (9)-(14)

$/ *$ Calculate the current state $x(t)=\left\{\left(x_{N}(t), x_{I}(t), x_{R}(t), x_{M}(t)\right\}\right.$ and costate values of the node, $\lambda_{D}$ and $\lambda_{A} * /$

Calculate the defender's payoff $g_{D}\left(t, x(t), P_{A}(t), P_{D}(t)\right)$

Calculate the attacker's payoff $g_{A}\left(t, x(t), P_{A}(t), P_{D}(t)\right)$

Calculate the optimal strategies of the defender and attacker, $p_{D}(t)^{*}$ and $p_{A}(t)^{*}$

Calculate the result of the attack-defense confrontation, $\tau_{N I}, \tau_{N R}, \tau_{I N}, \tau_{I R}$, and $\tau_{I M}$

ENDFOR

Return $p_{D}(t)^{*}$

END

Algorithm 1: Algorithm to select optimal defense strategy in attack-defense differential game.

TABLE 1: Comparison of models and methods.

\begin{tabular}{|c|c|c|c|c|c|c|}
\hline Literature & Game type & $\begin{array}{l}\text { Player } \\
\text { type }\end{array}$ & $\begin{array}{c}\text { Real-time performance of } \\
\text { decision-making }\end{array}$ & $\begin{array}{c}\text { Random } \\
\text { disturbance }\end{array}$ & $\begin{array}{l}\text { Equilibrium } \\
\text { solution }\end{array}$ & Application \\
\hline [12] & Single-stage game & 2 & - & No & Simple & Strategy selection \\
\hline$[14]$ & Multistage game & 2 & Poor & No & Detailed & $\begin{array}{l}\text { Intrusion } \\
\text { detection }\end{array}$ \\
\hline [19] & Differential game & 2 & Real-time & No & Detailed & Strategy selection \\
\hline [21] & Differential game & 2 & Real-time & No & Simple & Strategy selection \\
\hline [24] & Stochastic game & 2 & Poor & Yes & Simple & Safety analysis \\
\hline [25] & Stochastic game & 2 & Poor & Yes & Detailed & $\begin{array}{l}\text { Situation } \\
\text { assessment }\end{array}$ \\
\hline This paper & $\begin{array}{c}\text { Stochastic differential } \\
\text { game }\end{array}$ & $n$ & Real-time & Yes & Detailed & Strategy selection \\
\hline
\end{tabular}

TABLE 2: Description of attack actions.

\begin{tabular}{|c|c|c|c|c|}
\hline No. & Attack action & Attack strength & Attack type & Average strength \\
\hline 1 & Install trojan & 0.9 & & \\
\hline 2 & Attack SSH on web server & 0.82 & $A_{H}$ & 0.82 \\
\hline 3 & Install listener program & 0.74 & & \\
\hline 4 & Send abnormal data to buffer & 0.5 & & \\
\hline 5 & LPC to LSASS process & 0.45 & $A_{M}$ & 0.45 \\
\hline 6 & SQL injection & 0.4 & & \\
\hline 7 & FTP host attack & 0.35 & & \\
\hline 8 & Apache chunk overflow & 0.3 & $A_{L}$ & 0.3 \\
\hline 9 & Oracle TNS listener & 0.25 & & \\
\hline
\end{tabular}

The attack-defense confrontation is simulated for $\delta_{D}=\delta_{A}=0 ; \delta_{D}=0.01, \delta_{A}=0.03$; and $\delta_{D}=0.06, \delta_{A}=0.08$. The experimental results are shown in Figures $1-4$.

4.2.1. Analysis of Number of Transitioned Nodes in Different Security Sates. Figure 1 shows plots of the trends of the number of nodes in all states with time under different random intensity disturbances. The horizontal axis is time $t$. The $N, I, R$, and $M$ curves on the vertical axis indicate the number of nodes in the normal, infected, restored, and malfunctioned states, respectively. 
TABle 3: Description of defense actions.

\begin{tabular}{|c|c|c|c|c|}
\hline No. & Defense action & Defense strength & Defense type & Average strength \\
\hline 1 & Increase password complexity & 0.8 & \multirow{3}{*}{$D_{H}$} & \multirow{3}{*}{0.76} \\
\hline 2 & Uninstall delete trojan & 0.8 & & \\
\hline 3 & Change account and password & 0.7 & & \\
\hline 4 & Restart FTP server & 0.6 & \multirow{4}{*}{$D_{M}$} & \multirow{4}{*}{0.5} \\
\hline 5 & Renew root data & 0.5 & & \\
\hline 6 & Correct homepage & 0.5 & & \\
\hline 7 & Code randomization & 0.4 & & \\
\hline 8 & Identify code injection & 0.3 & \multirow{3}{*}{$D_{L}$} & \multirow{3}{*}{0.267} \\
\hline 9 & Add physical resource & 0.3 & & \\
\hline 10 & Delete suspicious account & 0.2 & & \\
\hline
\end{tabular}

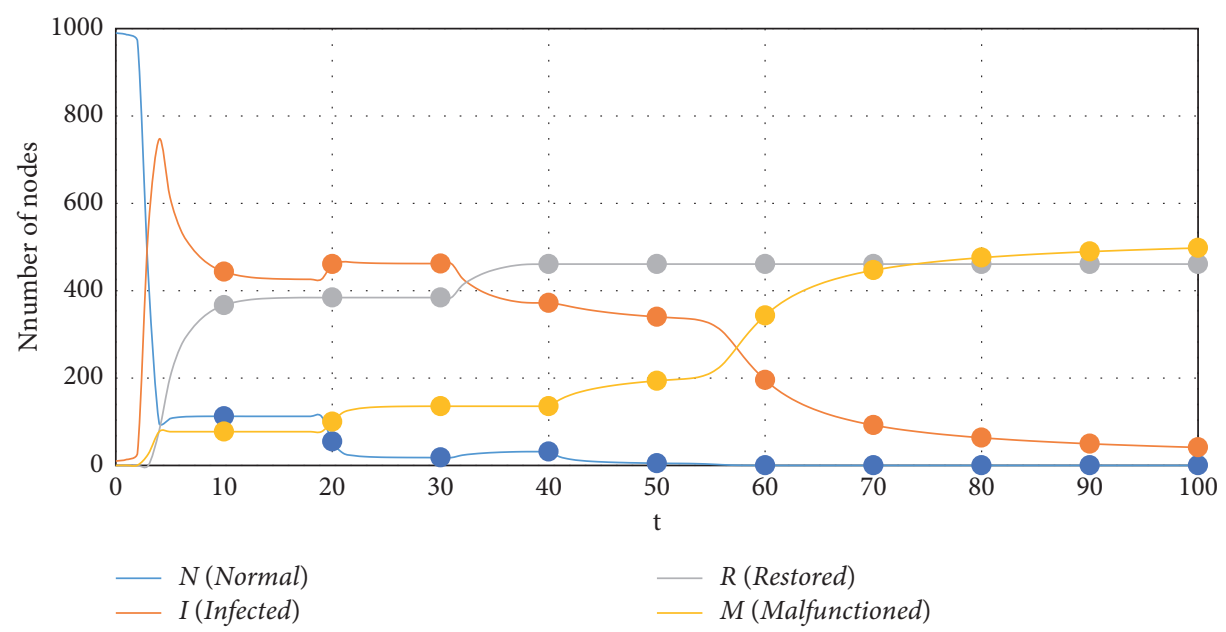

(a)

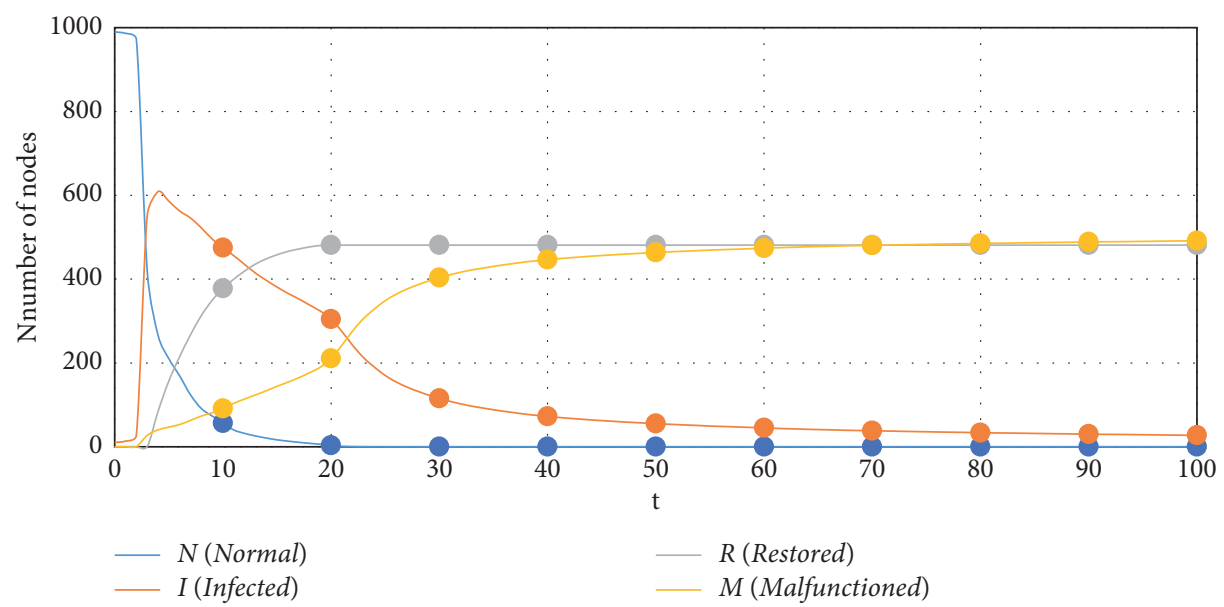

(b)

Figure 1: Continued. 


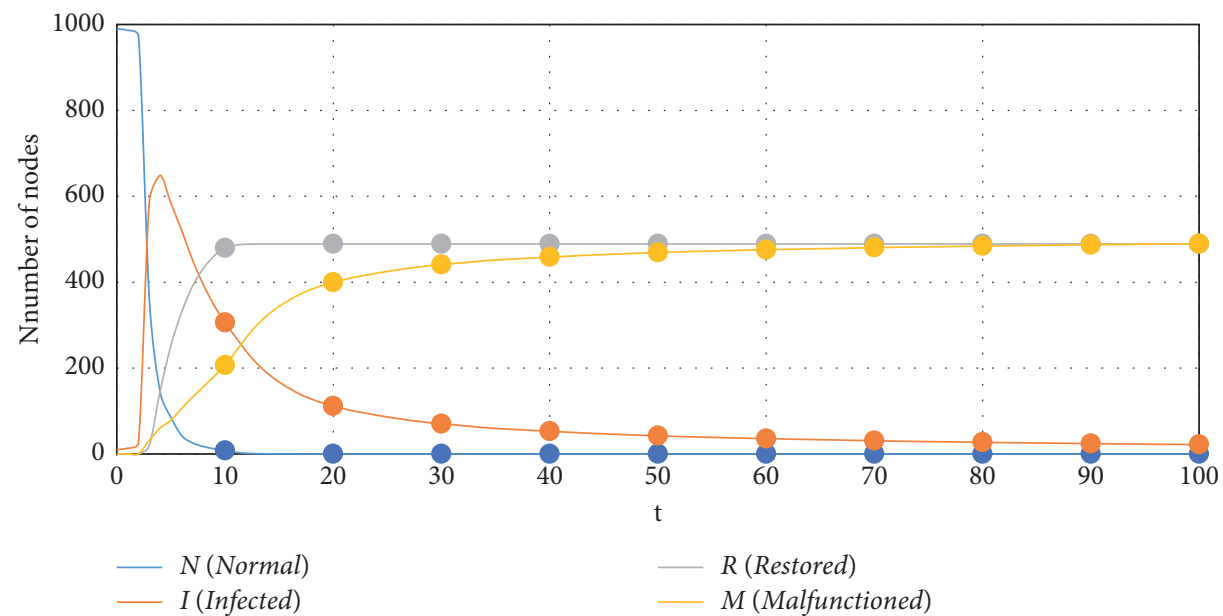

(c)

Figure 1: The numbers of nodes in different security states. (a) $\delta_{D}=\delta_{A}=0$. (b) $\delta_{D}=0.01, \delta_{A}=0.03$. (c) $\delta_{D}=0.06, \delta_{A}=0.08$.

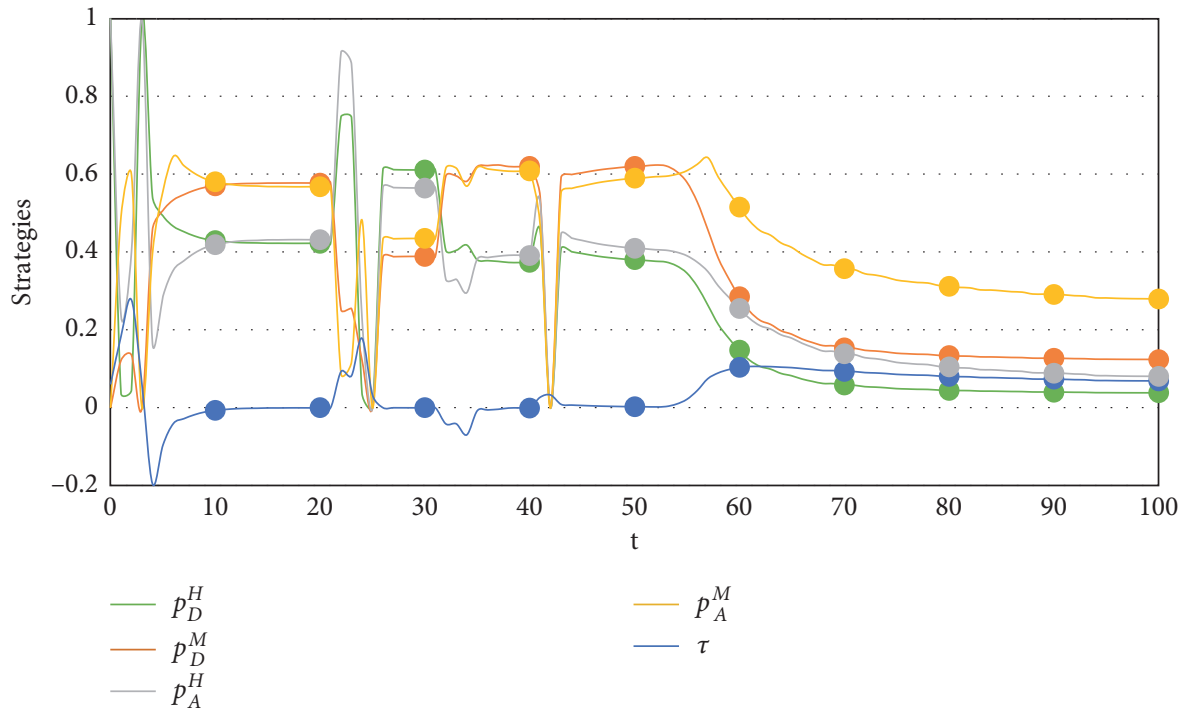

Figure 2: The trajectories of attack and defense strategies when $\delta_{D}=\delta_{A}=0$.

(1) Some common points in the above three figures are observed. In the early stages of network attack-defense confrontation, normal nodes can quickly transition to the infected state. At about $t=4.7 \mathrm{~s}$, the number of infected nodes reaches the maximum, because, in the initial stage of attack-defense confrontation, there are many normal nodes, with high strategy execution costs, while the number of infected nodes is small, with relatively low-strategy execution costs. So, the attacker will choose a high-intensity attack strategy, while the defender is subject to cost constraints and cannot respond to the attack strategy in time, resulting in the infection of a large number of normal nodes. Similarly, when the number of infected nodes is large, the defender can transition some of the infected nodes to the normal and restored states. As the game progresses, the number of nodes will eventually approach 500 in both the restored and malfunctioned states, and the number in the infected and malfunctioned states will eventually approach zero.

(2) It can be seen from the above figures that, for random disturbance intensities of $\delta_{D}=\delta_{A}=0$, the curves of changes of the numbers of nodes tend to level off after $t=66 \mathrm{~s}$; for $\delta_{D}=0.01$ and $\delta_{A}=0.03$, these curves tend to level off after $t=33 \mathrm{~s}$; and for $\delta_{D}=0.06$ and $\delta_{A}=0.08$, they tend to level off after $t=23 \mathrm{~s}$. Therefore, when the intensity of random disturbance increases, the time required for the number of nodes to stabilize decreases. The reason is as follows. After a normal node is infected, the attacker will quickly turn it into a malfunctioned state, so that the number of infected nodes can be kept at a relatively low level, and the cost of executing the attack is lower. However, due to the 


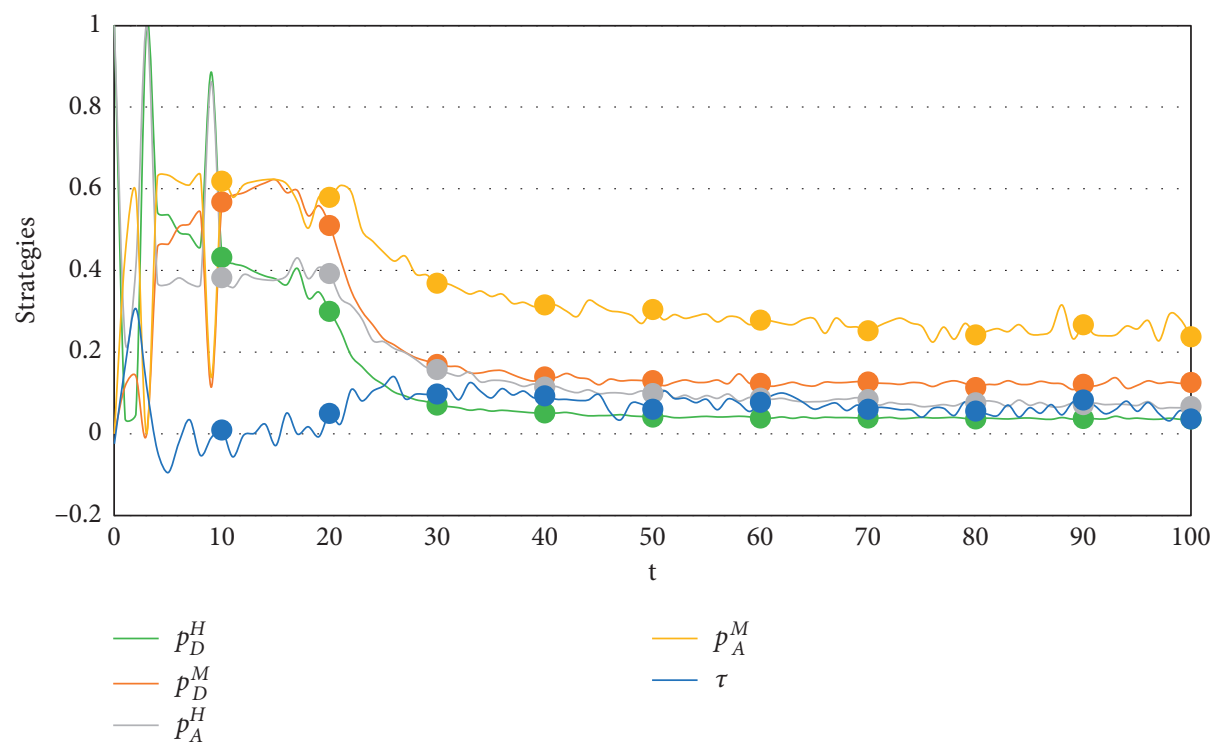

Figure 3: The trajectories of attack and defense strategies when $\delta_{D}=0.01, \delta_{A}=0.08$.

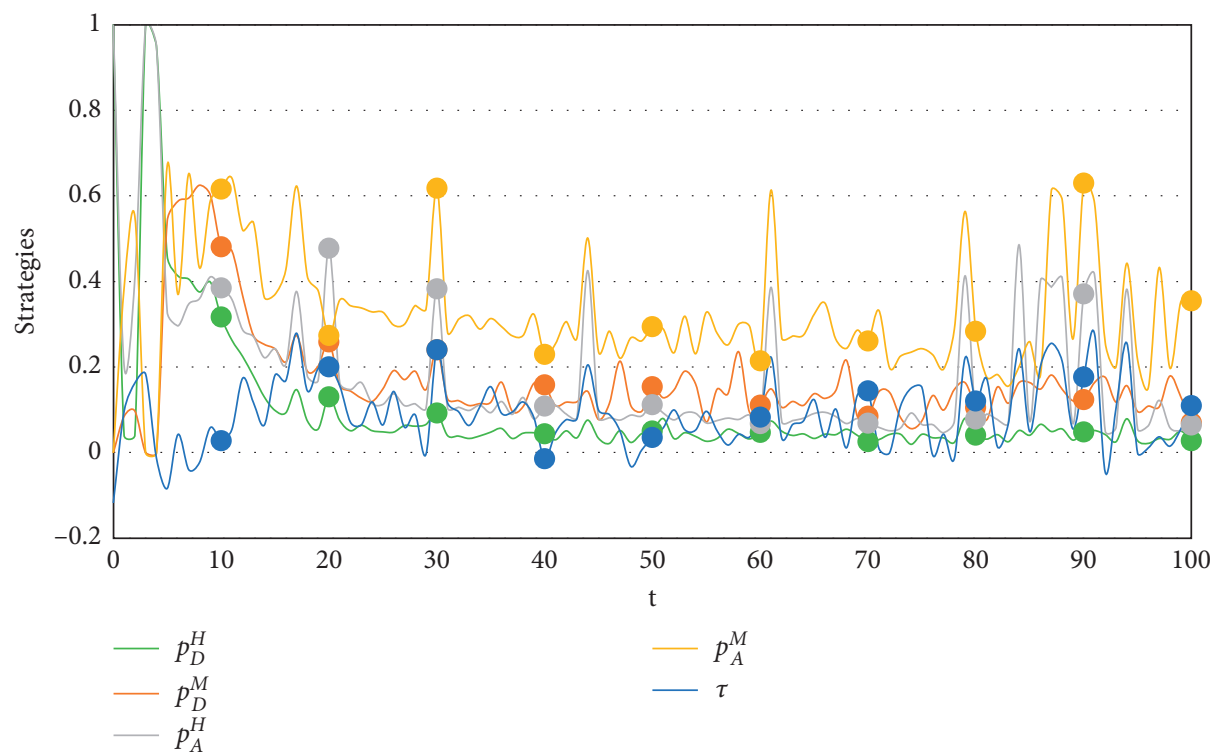

FIgURE 4: The trajectories of attack and defense strategies when $\delta_{D}=0.06, \delta_{A}=0.08$.

large number of nodes on the defender side, defense strategy execution cost is relatively high. The attacker eventually has stronger antidisturbance ability. Therefore, when the intensity of random disturbance increases, the node infection rate increases, and it takes less time for the game to reach a steady state.

(3) The following can be found from the above analysis. The influence of random factors can speed up the network attack-defense process, but the final numbers of various nodes are not changed much by random disturbances, indicating that the model has a certain antidisturbance ability. Random disturbances have a great impact on the game process. The defender should reduce these as much as possible to ensure that network changes do not deviate from its own expectations. The attacker and defender are constrained by costs. When one side has a large number of nodes, a strategy will often incur more costs, providing opportunity to the other side. Therefore, the defender should take measures to reduce defense costs, in order to preserve more normal nodes in the confrontation.

4.2.2. Trajectory Analyses of Optimal Dynamic Strategies of the Attacker and Defender. Figures 2-4 show the trajectory of the dynamic strategies of the attacker and defender over 
time under random disturbances of different intensities. The horizontal axis represents time $t$. The vertical axes $p_{D}^{H}(t)$ and $p_{D}^{M}(t)$, respectively, represent the probabilities that the defender chooses high- and medium-intensity defense strategies. $p_{A}^{H}(t)$ and $p_{A}^{M}(t)$, respectively, represent the probabilities of the attacker choosing high- and mediumintensity attack strategies. $\tau(t)=a_{\text {act }}(t)-d_{\text {act }}(t)$ represents the result of the attack-defense confrontation with $\tau(t) \geq 0$ representing a successful attack, and $\tau(t)<0$ represents a successful defense.

(1) According to Figure 2, the game can be divided into stages of defense, stalemate, and penetration. The defense stage occurs at $t \in[0,7]$, when the attacker and defender tend to adopt high-intensity strategies, and the game process is more intense. The attacker tries to use a high-intensity strategy in the early stage of the game to cause as much loss as possible to the defender and transitions some normal nodes into the infected state. The defender uses a high-intensity strategy to reduce losses and turns some nodes into the restored state. After a period of time, due to the high execution costs of high-intensity strategies, the attacker and defender reduce the probabilities of using them. The attacker and defender enter a stalemate stage at $t \in[7,63]$, both adopt medium-intensity strategies, and both can achieve a balance between attack and defense. At $t \in[22,26]$, the attacker attempts a high-intensity attack. Although the defender adopts certain countermeasures, it is successfully attacked, and some normal nodes are infected. After $t=63 \mathrm{~s}$, the game enters the penetration stage, in which the attacker and defender tend to adopt low-intensity strategies, because it is difficult for either to reverse the current situation, and low-intensity strategies can reduce costs. However, the attacker has the advantage of a higher overall cost-effectiveness ratio, and infected nodes will gradually turn into the malfunctioned state.

(2) It can be seen from Figure 3 that, in the case of relatively weak random disturbances, the trajectories of the dynamic strategies of the attacker and defender are similar to those without random disturbance. The defense stage exists at $t \in[0,7]$, the stalemate stage at $t \in[7,26]$, and the penetration stage at $t>26 \mathrm{~s}$. The main intensities of strategies in the defense, stalemate, and penetration stages are high, medium, and low, respectively. However, under the influence of random factors, the elapsed time of stalemate is significantly reduced, from $56 \mathrm{~s}$ to $19 \mathrm{~s}$. In this stage, the two sides have achieved certain results. The attacker turns the infected nodes into the malfunctioned state, and the defender turns the normal nodes into the restored state.

(3) According to Figure 4, when the random disturbances are strong, the strategy trajectories of the attacker and defender change significantly, but the three stages of the game still exist. The stalemate stage exists at $t \in[7,17]$. It can be found that the increase of random disturbances has the greatest impact on the stalemate stage of the game process, whose time noticeably shortens. In the stalemate stage, the defender always tends to adopt a conservative strategy, i.e., turning the normal nodes into the restored state to ensure their safety, rather than restoring the infected nodes to reduce losses. During the game process, the attacker turns the infected nodes into the malfunctioned state. As a result, in the penetration stage, the number of restored nodes is always larger, while the number of infected nodes is smaller, so that the attacker can rely on the cost advantage to gradually turn the infected nodes to the malfunctioned state.

(4) Figures 2-4 and the above analysis indicate that, in the stalemate stage, the defender mainly adopts a medium-intensity strategy. However, the stalemate stage has a great impact in the entire game process and lasts a long time. In this stage, the attacker may adopt a surprise attack strategy. Therefore, the defender must try to increase the cost-effectiveness ratio of the medium-intensity strategy to ensure its advantage in the stalemate stage and try to achieve the maximum defense effectiveness at the minimum cost. The defender should also increase the upper limit of the strategy strength as much as possible, reduce the gap between it and the attacker, and reduce the loss when defense is impossible.

\section{Conclusion}

The actual network attack and defense confrontations often occur continuously and in real time and are affected by random disturbance factors. Dynamic network attack and defense analysis models based on game theory mainly include staged and differential game models. The staged game model cannot meet the requirements of network realtime decision-making, and the differential game model does not consider uncertain factors in the continuous realtime attack-defense process. Therefore, it is difficult to meet the requirements of real-time defense decision-making in network attack and defense scenarios with random disturbances. This study considers random disturbances in network attack-defense confrontations, describes the transition process of the network security states by differential equations, constructs a network attack-defense stochastic differential game model, and proposes a method to select the optimal defense strategy based on real-time analysis of network attack-defense confrontation under the influence of random disturbances. The simulation experiment verifies the effectiveness and rationality of the proposed model and method, which can provide a reference for the selection of active defense strategy under random disturbances. 


\section{Data Availability}

The experimental code and associated data have been uploaded to GitHub at https://github.com/kaster-hn/ Network-attack-defense-stochastic-differential-gamealgorithm.

\section{Conflicts of Interest}

The authors declare that they have no conflicts of interest regarding the publication of this paper.

\section{Authors' Contributions}

Yan Mi and Hengwei Zhang contributed equally to this work.

\section{Acknowledgments}

This work was supported by the National Key Research and Development Program of China under Grant no. 2017YFB0801900 and the National Natural Science Foundation of China under Grant no. 61902427.

\section{References}

[1] L. Shang, W. Zhao, J. Zhang, Q. Fu, Q. Zhao, and Y. Yang, "Network security situation prediction based on long shortterm memory network," in Proceedings of the 2019 20th AsiaPacific Network Operations and Management Symposium (APNOMS), pp. 1-4, Matsue, Japan, September 2019.

[2] Amr, Kaspersky Security Bulletin 2019. Statistics, Kaspersky, Moscow, Russia, 2019.

[3] H. Hu, W. Ma, and W. Luo, "A method for detecting largescale network anomaly behavior," ITM Web of Conferences, vol. 17, pp. 1012-1020, 2018.

[4] X. Liu, H. Zhang, Y. Zhang, L. Shao, and J. Han, "Active defense strategy selection method based on two-way signaling game," Security and Communication Networks, vol. 2019, Article ID 1362964, 14 pages, 2019.

[5] M. Maschler, Game Theory, Cambridge University Press, Cambridge, UK, 2013.

[6] H. Hu, Y. Liu, C. Chen, H. Zhang, and Y. Liu, "Optimal decision making approach for cyber security defense using evolutionary game," IEEE Transactions on Network and Service Management, vol. 17, no. 3, pp. 1683-1700, 2020.

[7] Y. Hayel and Q. Zhu, "Epidemic protection over heterogeneous networks using evolutionary Poisson games," IEEE Transactions on Information Forensics and Security, vol. 12, no. 8, pp. 1786-1800, 2017.

[8] Z. Tian, X. Gao, S. Su, J. Qiu, X. Du, and M. Guizani, "Evaluating reputation management schemes of internet of vehicles based on evolutionary game theory," IEEE Transactions on Vehicular Technology, vol. 68, no. 6, pp. 5971-5980, 2019.

[9] J.-1. Tan, C. Lei, H.-q. Zhang, and Y.-q. Cheng, "Optimal strategy selection approach to moving target defense based on Markov robust game," Computers \& Security, vol. 85, no. 5, pp. 63-76, 2019.

[10] Y. Yang, B. Che, Y. Zeng, Y. Cheng, and C. Li, "MAIAD: a multistage asymmetric information attack and defense model based on evolutionary game theory," Symmetry, vol. 11, no. 2, pp. 215-229, 2019.
[11] S. Sarıtaş, S. Gezici, and S. Yüksel, "Hypothesis testing under subjective priors and costs as a signaling game," IEEE Transactions on Signal Processing, vol. 67, no. 19, pp. 5169-5183, 2019.

[12] A. Aydeger, M. H. Manshaei, M. A. Rahman, and K. Akkaya, "Strategic defense against stealthy link flooding attacks: a signaling game approach,” 2019, https://arxiv.org/abs/1912. 10073.

[13] X. Chen, X. Liu, L. Zhang, and C. Tang, "Optimal defense strategy selection for spear-phishing attack based on a multistage signaling game," IEEE Access, vol. 7, pp. 19907-19921, 2019.

[14] S. Shen, L. Huang, H. Zhou, S. Yu, E. Fan, and Q. Cao, "Multistage signaling game-based optimal detection strategies for suppressing malware diffusion in fog-cloud-based IoT networks," IEEE Internet of Things Journal, vol. 5, no. 2, pp. 1043-1054, 2018.

[15] X. Li, Y. Lu, S. Liu, and W. Nie, "Network security situation assessment method based on Markov game model," TIIS, vol. 5, no. 125, pp. 2414-2428, 2018.

[16] D. Sahabandu, J. Allen, S. Moothedath, L. Bushnell, W. Lee, and R. Poovendran, "Quickest detection of advanced persistent threats: a semi-markov game approach," in Proceedings of the IEEE 11th International Conference on Cyber-Physical Systems (ICCPS), pp. 9-19, Sydney, Australia, April 2020.

[17] H. Li, W. Shen, and Z. Zheng, "Spatial-temporal moving target defense: a Markov Stackelberg game model," 2020, https://arxiv.org/pdf/2002.10390.

[18] F. A. Faruqi, Differential Game Theory with Applications to Missiles and Autonomous Systems Guidance, Wiley, Hoboken, NJ, USA, 2017.

[19] Y. Huang and Q. Zhu, "A differential game approach to decentralized virus-resistant weight Adaptation policy over complex networks," IEEE Transactions on Control of Network Systems, vol. 7, no. 2, pp. 944-955, 2020.

[20] M. Li and S. Li, "A differential game-theoretic approach for the intrusion prevention systems and attackers in wireless networks," Wireless Personal Communications, vol. 103, no. 3, pp. 1993-2003, 2018.

[21] G. Feng, J. Lin, Q. Zhao et al., "A differential game based approach against objective function attack in cognitive networks," Chinese Journal of Electronics, vol. 27, no. 4, pp. 879-888, 2018.

[22] L.-X. Yang, P. Li, Y. Zhang, X. Yang, Y. Xiang, and W. Zhou, "Effective repair strategy against advanced persistent threat: a differential game approach," IEEE Transactions on Information Forensics and Security, vol. 14, no. 7, pp. 1713-1728, 2019.

[23] J. Ren and C. Zhang, "A differential game method against attacks in heterogeneous honeynet," Computers \& Security, vol. 97, Article ID 101870, 2020.

[24] H. Orojloo and M. A. Azgomi, "A stochastic game model for evaluating the impacts of security attacks against cyberphysical systems," Journal of Network and Systems Management, vol. 26, no. 4, pp. 929-965, 2018.

[25] J. Liu, F. Weng, R. Zhang, and Y. Guo, "Network security situation assessment approach based on attack-defense stochastic game model," in Proceedings of the International conference on cloud computing and security (ICCCS 2018), pp. 161-173, Beijing, China, June 2018.

[26] E. M. Kandoussi, M. Hanini, I. El Mir, and A. Haqiq, "Toward an integrated dynamic defense system for strategic detecting attacks in cloud networks using stochastic game," Telecommunication Systems, vol. 73, no. 3, pp. 397-417, 2020. 
[27] H. Zhang and T. Li, "Optimal active defense based on multistage attack-defense signaling game," Acta Electronica Sinica, vol. 45, no. 2, pp. 431-439, 2017.

[28] Scalable simulation framework. [Online]. Available: http:// www.ssfnet.org/homePage.html.

[29] S. Yoon and Y. Kim, "A design of network simulation environment using ssfnet," in Proceedings of the 2009 First International Conference on Advances in System Simulation, pp. 73-78, IEEE, Porto, Portugal, September 2009.

[30] W. Qi, Y. Luo, D. Fang, and X. Chen, "Simulation of antiworm counter-attack based on SSFNet," Computer Engineering, vol. 33, pp. 149-152, 2007.

[31] M. Pendleton, R. Garcia Lebron, J. H. Cho, and S. Xu, "A survey on systems security metrics," ACM Computing Surveys, vol. 49, no. 4, p. 62, 2017.

[32] L. Gordon, M. Loeb, W. Lucyshyn, and R. Richardson, "CSI/ FBI computer crime and security survey," Proceedings of the Computer Security Institute, San Francisco, CA, USA, 2015. 